Sportwiss 2011 · 41:190-201 DOI 10.1007/s12662-011-0195-7

Online publiziert: 6 . August 2011

(c) Springer-Verlag 2011
Mirko Schmidt · Achim Conzelmann

Institut für Sportwissenschaft (ISPW), Universität Bern

\title{
Selbstkonzeptförderung im Sportunterricht
}

\section{Eine psychologische Betrachtung einer pädagogischen Zielperspektive}

Für die Legitimation des Schulsports finden sich immer wieder sportpädagogische Postulate, die die positive Wirkung geeigneter sportlicher Aktivitäten auf die Persönlichkeitsentwicklung betonen. So ist beispielsweise im gemeinsam von der Deutschen Vereinigung für Sportwissenschaft (dvs), dem Deutschen Sportlehrerverband (DSLV) und dem Deutschen Olympischen Sportbund (DOSB) beschlossenen Memorandum zum Schulsport zu lesen, dass der Schulsport grundsätzlich „,die (doppelte) Aufgabe [habe], sowohl die Sport- und Bewegungskultur zu erschließen als auch die Persönlichkeit zu entwickeln“" (dvs, DSLV \& DOSB, 2009, S. 5). Ähnliche Begründungslinien lassen sich international nahezu beliebig viele finden. Sportpsychologie und Sportpädagogik tun sich aber noch immer schwer, empirische Belege für dieses sportpädagogische Postulat zu finden.

Für Traitansätze (z. B. Big Five; Costa \& McCrae, 1992) scheint dies kaum verwunderlich, da diese eine hohe zeitliche Stabilität aufweisen und es daher wenig Sinn macht, durch kurz- bis mittelfristige Interventionen Veränderungen dieser Persönlichkeitsmerkmale herbeiführen zu wollen. Seit aber die kognitive Wende in der Psychologie auch die Sportwissenschaft erreicht hat, ist in der sportwissenschaftlichen Persönlichkeitsforschung eine Ausrichtung auf weniger stabile und damit plastischere selbst- und umweltbezogene Kognitionen festzustellen (Conzelmann, 2001, 2009). Diese Ausrichtung hat mehrere sportpädagogisch ausgerichtete Arbeitsgruppen dazu bewogen, der Forderung nach empirischen Nachweisen für den schulischen (oder außerschulischen) Sport nachzukommen (z. B. Brettschneider \& Kleine, 2002; Burrmann, 2004; Gerlach, 2008; Heim, 2002; Neuber, 2007; Sygusch, 2007; Tietjens, 2009). Dass dabei, ähnlich wie in der entwicklungspsychologischen Forschung, eine Fokussierung auf das Selbstkonzept stattgefunden hat (Greve, 2000; Krampen, 2002), lässt sich in mehrerlei Hinsicht begründen: Das Selbstkonzept, verstanden als selbstbezogenes Wissenssystem (Filipp \& Mayer, 2005, S. 260), ermöglicht erstens bedingt durch dessen höhere Plastizität einen gegenstandsadäquateren Zugang zur Überprüfung des oben genannten pädagogischen Postulats. Zweitens wird in einem zeitgemäßen dynamisch-interaktionistischen Verständnis von Persönlichkeit die Rolle des Individuums als Gestalter seiner eigenen Entwicklung hervorgehoben. Drittens kommt dem Selbstkonzept deshalb eine große Bedeutung zu, weil es in vielen Lebensbereichen eine verhaltensregulative Funktion einnimmt (Marsh \& Hau, 2003; Roebers, 2007; Schütz \& Sellin, 2003). So hängt es beispielsweise vom Selbstkonzept der eigenen Fähigkeiten $\mathrm{ab}$, welche Ziele man sich setzt und welche sozialen Verpflichtungen man eingeht (Montada, 2008), welche akademischen Leistungen man erbringt (Marsh, Byrne \&
Yeung, 1999) oder wie zufrieden man mit dem gewählten Beruf ist (Judge \& Bono, 2001). Neben der Verbreitung von Selbstkonzeptansätzen in der wissenschaftlichen Auseinandersetzung mit veränderbaren Persönlichkeitsmerkmalen hat sich eine ähnliche Hinwendung auch in einigen Sportlehrplänen bemerkbar gemacht, indem - beispielsweise in MecklenburgVorpommern, Schleswig-Holstein oder Hamburg - gefordert wird, dass die Schülerinnen und Schüler ein positives Selbstkonzept ausbilden sollen.

Die pädagogische Frage nach dem anzusteuernden Ziel, dem Entwicklungssoll wird dadurch jedoch noch nicht beantwortet. Gerade die normative Begründung von Erziehungs- und Entwicklungszielen kann und soll aus einer pädagogischen Perspektive und somit im schulischen Kontext nicht ausgeklammert werden (z. B. Prohl, 2006, 216-222; Neuber, 2007 , S. 28). Wenn es also um die positive Beeinflussung des Selbstkonzepts im Sportunterricht geht, ist zu fragen, wohin sich Merkmale des Selbstkonzepts von Schülerinnen und Schülern entwickeln sollen. Was meint man, wenn man von einem positiven Selbstkonzept spricht? Geht es darum, ein möglichst hohes Selbstkonzept auszubilden oder gibt es weitere angemessene Zielperspektiven? Die aus unterschiedlichen Forschungszweigen stammenden Empfehlungen sind hierbei uneinheitlich, da sie (a) von einer möglichst realitätsangemessenen Selbstwahrnehmung als „Voraussetzung (...) für eine gesunde Persönlichkeitsentwick- 
lung" (Hurrelmann, 2006, S. 38), über (b) die Forderung nach einem „positiv-realistischen Selbstkonzept" (Sygusch, 2008, S. 143), bis hin zu (c) einer Erhöhung des Selbstkonzepts durch den Sportunterricht (Lintunen, 1999, S. 117; Stiller \& Alfermann, 2007, S. 136) reichen. Diese Empfehlungen gründen zwar auf evidenten Argumenten, sie entbehren jedoch zum einen einer explizit theoretischen Auseinandersetzung mit der Funktionalitätsfrage. Zum anderen erfolgt deren Beantwortung ohne Berücksichtigung vorliegender empirischer Befunde. Angesichts dieser Antinomie erstaunt, dass alle bis dato gefundenen Interventionsstudien im Sportunterricht auf eine Erhöhung des Selbstkonzepts der Schülerinnen und Schüler abzielen (Annesi, 2007; Calfas \& Cooper, 1996; Goni \& Zulaika, 2000; Lloyd \& Fox, 1992; Marsh \& Peart, 1988; Percy, Dziuban \& Martin, 1981; Petrakis \& Bahls, 1991; Smith, 1982) und dabei die Veridikalität, verstanden als den Grad der „Realitätsangemessenheit der Selbstwahrnehmung eigener Fähigkeiten und Leistungen" (Helmke, 1992, S. 197), außer Acht lassen. ${ }^{1}$ Wenn dann durch Querschnittsstudien gestützt gefolgert wird, dass Personen mit hohem Selbstkonzept bessere Leistungen bringen als Personen mit niedrigem, und daraus geschlossen wird, dass hohe Selbstkonzepte funktional seien, scheint dies schon deshalb problematisch, da es ja durchaus sein kann, dass Personen hohe Selbstkonzepte aufweisen, weil sie in einer entsprechenden Domäne hohe Fähigkeiten besitzen. In diesem Fall wäre das

\footnotetext{
1 Ein breit angelegtes Begriffsverständnis bestimmt Veridikalität als Grad, mit dem „Urteile mit den,tatsächlichen', d. h. den objektiv gemessenen Merkmalen und Merkmalsausprägungen übereinstimmen" (Schrader, 2006, S. 95). Die Auseinandersetzung mit der Frage nach der Urteilsgenauigkeit brachte dabei einen eigenen Forschungsansatz hervor (Funder, 1995), der sich mit Bedingungen und Konsequenzen akkurater Urteile beschäftigte. Ein Spezialfall akkurater Urteilsbildung ist derjenige der Selbstbeurteilung oder Selbsteinschätzung. Dabei wird das eigene Subjekt zum Objekt, über welches geurteilt wird. Helmkes (1992) enges Begriffsverständnis der Veridikalität als „Realitätsangemessenheit der Selbstwahrnehmung" (S. 197) grenzt somit den aus der Urteilsforschung stammenden Begriff weiter ein, um inn auf den Akt der Selbstwahrnehmung zu beschränken.
}

Selbstkonzept hoch, aber dennoch realitätsangemessen. Neben der Funktionalität der Höhe gilt es also auch die Funktionalität der Realitätsangemessenheit des Selbstkonzepts zu diskutieren. Der folgende Beitrag behandelt daher die Frage, welche Selbstkonzeptausprägungen aus einer psychologischen Perspektive durch den Sportunterricht gefördert werden sollen. Dazu werden theoretische Überlegungen, vorwiegend aber empirische Studien referiert, aus denen sich Empfehlungen für selbstkonzeptfördernde Interventionen im Sportunterricht ableiten lassen.

\section{Welche Selbstkonzeptausprä- gungen sind funktional?}

Obwohl sich Forscher unterschiedlichster psychologischer Teildisziplinen und Forschungstraditionen dem Problem der Funktionalität von Selbsteinschätzungen angenommen haben, gibt es bis heute keine integrative Theorie realitätsangemessener Selbstkonzepte (Wilson, 2009). Diskussionen mit mehr oder weniger konsistenten Ergebnissen wurden jedoch in der Sozial- und Motivationspsychologie (Selbstkonzept, Attributionen), in der Entwicklungspsychologie (Selbstkonzeptentwicklung) und in der Klinischen Psychologie (Depression) geführt (Helmke, 1992, S. 197). Da dabei allerdings die Frage nach der Funktionalität realitätsangemessener oder -ferner Selbstkonzepte nie explizit gestellt wurde, scheint es sinnvoll zu sein, den Blick etwas zu weiten und $\mathrm{zu}$ fragen, welche Selbsteinschätzungen denn funktional sind. Dies ermöglicht unter anderem den Einbezug von Überlegungen und Untersuchungen, die sich mit Selbstwirksamkeitserwartungen, ${ }^{2}$ als Selbstkonzepte der eigenen Wirksamkeit (Mummendey, 2006, S. 187) und mit positiven Illusionen, als „selbstwertdienliche

\footnotetext{
2 Bong und Skaalvik (2003), welche die beiden oft als distinkt dargestellten Konstrukte Selbstkonzept und Selbstwirksamkeit vergleichend diskutieren, kommen zum Schluss, dass die Gemeinsamkeiten die Unterschiede nahezu aufwiegen. So seien beiden Konstrukten die Zentralität der wahrgenommenen Kompetenz, die mehrdimensionale und hierarchische Struktur, die Inhaltsspezifität und die Vorhersagbarkeit von Motivation, leistungsfördernder Emotion und Leistung gemeinsam.
}

Selbsteinschätzungen", auseinandersetzen. Der Mangel an einschlägigen sportwissenschaftlichen Publikationen zur Funktionalitätsfrage führt dazu, dass sich diese Auseinandersetzung ausschließlich auf das Rezipieren von Studien der genannten psychologischen Teildisziplinen beschränken muss. Im Folgenden werden vorliegende Erkenntnisse zur Funktionalität einer Überschätzung, einer realistischen Einschätzung und einer Unterschätzung eigener Fähigkeiten und Persönlichkeitseigenschaften dargestellt.

\section{Selbstüberschätzung}

Bandura (1994) spricht sich in seiner Selbstwirksamkeitstheorie deutlich für den funktionalen Wert einer Überschätzung der eigenen Fähigkeiten aus. Er sieht in optimistischen, leicht überzogenen Selbstwirksamkeitserwartungen das Potenzial, den Menschen zu Taten zu bewegen, die er durch eine allzu akkurate Selbsteinschätzung unterlassen hätte. So sei das Leben voll von Unwegsamkeit, Hindernissen, Rückschlägen und Frustrationen, die nur mit entsprechender Selbstüberzeugung und der damit einhergehenden Leistungsmotivation zu meistern seien. Mit den Worten von Henry Ford könnte man sagen, dass wer immer nur das tut, was er schon kann, immer nur der sein wird, der er schon ist. Unterstützung erhält diese Position durch Befunde, die im Review von Taylor und Brown (1988) zusammengetragen wurden und in der Folge zu einer heftig ausgetragenen Debatte führten: die Optimismus-Realismus-Debatte. Taylor und Brown (1988) vertreten die These, dass unrealistisch positive Selbstbewertungen, übertrieben hohe Einschätzungen eigener Kontrolle (Kontrollillusion) und unrealistischer Optimismus sowohl zu psychischer Gesundheit als auch zu erhöhter Motivation, größerer Leistungsbereitschaft, somit besserer Leistung und schließlich zu mehr Erfolg führen. Ihren Ausführungen zufolge zeigen sich diese selbstwertdienlichen Selbsteinschätzungen in folgenden zwei Tatsachen: „(a) most individuals see themselves as better than the average person and (b) most individuals see themselves better than others see them" (Taylor \& Brown, 1988, 
S. 195-196). Diese bei den meisten gesunden Individuen vorzufindenden positiven Illusionen zeichnen sich insbesondere durch drei systematische Verzerrungen aus, die (a) die eigene Person, (b) die Welt und (c) die Zukunft betreffen (Taylor \& Brown, 1994, S. 21). Dass diese positiven Illusionen normal, adaptiv und gesund sein sollen, scheinen auch Befunde aus der Depressionsforschung zu bestätigen. Alloy und Abramson (1979) konnten zeigen, dass nichtdepressive Individuen häufiger optimistischen Verzerrungen unterliegen als depressive Personen. ${ }^{3}$ Ihr Fazit aus weiteren Studien, die durch die überraschend realitätsangemessene Selbsteinschätzung Depressiver angeregt wurden, war dabei folgendes: „The depressive's problem is not that he or she suffers from the presence of depressogenic cognitive biases, but rather that he or she suffers from the absence of the nondepressive cognitive biases" (Abramson \& Alloy, 1981, S. 445). ${ }^{4}$ Positive Illusionen scheinen demnach ein Schutzfaktor gegen Depression oder depressive Verstimmungen und ein generelles Kennzeichen psychischer Gesundheit zu sein (Taylor \& Brown, 1988). Angestoßen durch diese Erkenntnis wurde im Laufe der Jahre eine Vielzahl an Befunden zusammengetragen, welche die überwiegend positiven Effekte positiver Illusionen stützen (Armor \& Taylor, 1998; Bonanno, Field, Kovacevic \& Kaltman, 2002; Kurt \& Paulhus, 2008; Sedikides, Rudich, Gregg, Kumashiro \& Rusbult, 2004; Taylor \& Brown, 1994; Taylor \& Gollwitzer, 1995; Taylor, Kemeny, Reed, Bower \& Gruenewald, 2000; Tay-

\footnotetext{
3 In der sog. Kontingenzlernaufgabe ließen sie depressive und nichtdepressive Personen schätzen, in welchem Ausmaß sie das Aufleuchten eines Lichtes durch das eigene Betätigen einer Taste erklärten. Was den Versuchspersonen verschwiegen wurde: Das tatsächliche Ausmaß an Kontrolle über das Aufleuchten des Lichts wurde experimentell manipuliert. Dabei zeigte sich, dass nichtdepressive Probanden bei 75\%iger Übereinstimmung zwischen der Betätigung der Taste und dem Aufleuchten des Lichts ihren Einfluss auf dieses Ereignis massiv überschätzten, während depressive Probanden ein realistisches $\mathrm{Ma}$ an Kontrolle angaben.

4 Für einen kritischen Überblick zum Phänomen des Depressiven Realismus siehe z. B. Allan, Siegel und Hannah (2007) oder Carson, Hollon und Shelton (2010).
}

lor, Lerner, Sherman, Sage \& McDowell, 2003; Willard \& Gramzow, 2009; Wright, 2000).

\section{Realistische Selbsteinschätzung}

Trotz dieser scheinbar starken empirischen Evidenz der Funktionalität positiver Illusionen für die psychische Gesundheit wurde heftige Kritik an dieser Position und dem damit einhergehenden Menschenbild geübt. Colvin und Block (1994) betonen in der Tradition der humanistischen Psychologie (z. B. Rogers, 1951), dass nur eine realistische Selbstund Weltsicht die psychische und physische Gesundheit fördern könne - sogar dann, wenn realistisch mit negativ gleichgesetzt werden müsse. Sie stellen sich den von Taylor und Brown $(1988,1994)$ vorgetragenen Befunden mit methodologischer Kritik und führen ins Feld, die Akkuratheit der Realitätseinschätzungen sei nicht valide erhoben worden. Dabei fordern sie ein externales Validitätskriterium, das es ermöglicht, Personen mit positiver Selbsteinschätzung - die in realis eine Entsprechung finden - von jenen zu unterscheiden, denen diese Entsprechung fehlt. Die Funktionalitätsfrage beantworten zu wollen, indem sowohl die unabhängige als auch die abhängige Variable über ein selbstberichtetes Konstrukt operationalisiert wird, sei per se ein sinnloses Unterfangen. So könne davon ausgegangen werden, dass Personen, die sich selbst überschätzen, generell überzogene Selbstansichten haben und dass so auch das selbstberichtete Konstrukt (z. B. hohes Selbstwertgefühl oder subjektives Wohlbefinden - als abhängige Variable) einer positiven Verzerrung unterliegt (s. auch Shedler, Mayman \& Manis, 1993). Zudem bezweifeln Colvin und Block (1994, S. 14) die Übertragbarkeit von Erkenntnissen aus Laboruntersuchungen: Wenn es um die Bestimmung von möglichen Konsequenzen positiver Illusionen geht, fokussieren Laboruntersuchungen gezwungenermaßen kurzfristige Outputs, die mit den langfristigen Konsequenzen nicht übereinzustimmen brauchen. In ihrer Längsschnittstudie versuchen Colvin, Block und Funder (1995) diesen beiden Kritikpunkten Rechnung zu tragen, indem sie im Abstand von fünf Jahren sowohl den studentischen Probanden als auch vier zusätzlichen Versuchsleitern ein Kartenset mit Persönlichkeitseigenschaften unterbreiten und diese bitten, sich selbst bzw. die Probanden mit den Begriffen möglichst präzise zu beschreiben. Personen, die sich zum ersten Messzeitpunkt überschätzten, wurden zum zweiten Messzeitpunkt als zynisch, argwöhnisch, unsicher, dünnhäutig und misstrauisch beschrieben, während Personen, die sich realistisch einschätzten, als aufrichtig, direkt, ehrlich und ausgeglichen beschrieben wurden. Daraus folgern sie, dass eine Selbstüberschätzung, operationalisiert über ein externales Kriterium, besonders im sozialen Kontext negative Konsequenzen mit sich bringen kann. Ein breites Begriffsverständnis von psychischer Gesundheit müsse auch soziale Faktoren berücksichtigen, was ihrer Ansicht nach die zu große Beachtung findenden Ergebnisse von Taylor und Brown (1988) obsolet werden ließen.

Neben dieser eher methodologisch ausgerichteten Kritik an der Funktionalität überzogener Selbsteinschätzungen für das psychische Wohlbefinden des befragten Individuums wird ein zweiter Aspekt diskutiert, der sich auf den Einfluss der Selbstüberschätzung auf die Umwelt (namentlich auf mögliche Interaktionspartner) bezieht. Taylor und Brown (1988, S. 198) behaupten, dass hohe Selbsteinschätzungen auch mit der Fähigkeit einhergehen, sich um andere zu kümmern. Dies soll u. a. deshalb der Fall sein, weil optimistische Menschen generell besser in der Lage seien, gute Stimmung zu verbreiten, sich eher trauen würden, Lob auszusprechen und daher beliebtere Interaktionspartner seien. Gestützt wird diese These z. B. von einer Studie, die sich mit der Entstehung neuer Freundschaften bei Studienanfängern beschäftigt. So stellten Brissette, Scheier und Carver (2002, S. 105) fest, dass Optimisten (als zukunftsgerichtete Selbstüberschätzer) schon 2 Wochen nach Studienbeginn deutlich mehr Freunde gefunden hatten als Pessimisten und gegen Ende Semester einen größeren Freundeskreis hatten. Diesen Befunden steht jedoch eine Reihe von Untersuchungen entgegen, die eher von negativen sozialen Konsequenzen hoher Selbsteinschätzung be- 
richten und Selbstüberschätzer als problematische Interaktionspartner beschreiben, da sie sich durch mangelnde Empathie, hohe Narzissmuswerte und erhöhte Gewaltbereitschaft auszeichnen (Baumeister, Bushman \& Campbell, 200o; Baumeister, Campbell, Krueger \& Vohs, 2003; Bushman \& Baumeister, 1998; Gresham, Lane, MacMillan, Bocian \& Ward, 2000). Zudem wird Überschätzern zwar kurzfristig höhere soziale Akzeptanz zugestanden, die sich jedoch in einer eher längerfristigen Perspektive ins Gegenteil kehrt (Colvin et al., 1995; Paulhus, 1998; Robins \& Beer, 2001). So konnte Paulhus (1998) zeigen, dass Selbstüberschätzer in wöchentlich stattfindenden Gruppendiskussionen zu Beginn sehr hohe Akzeptanzwerte der anderen Teilnehmer erhielten, nach 7 Wochen aber sehr negativ beurteilt wurden. Dieser Abfall an sozialer Akzeptanz über die Zeit kann mit kompetitiven Selbstdarstellungstendenzen erklärt werden, die sich nicht als sozialverträglich erweisen. Neuere Untersuchungen scheinen diese kurzfristig positiven, aber langfristig negativen Auswirkungen von Selbstüberschätzung auf den interpersonalen Bereich zu erhärten (Anderson, Srivastava, Beer, Spataro \& Chatman, 2006; Bonanno, Rennicke \& Dekel, 2005; Church, Katigbak, Prado, Valdez-Medina, Miramontes \& Ortiz, 2006; Kwan, John, Robins \& Kuang, 2008).

Für die Funktionalität einer realistischen Selbsteinschätzung sprechen auch die Ergebnisse der modernen Attributionsforschung. Angetrieben durch das Grundbedürfnis, über die Ursachen von Ereignissen oder Ergebnissen eigener Handlungen informiert zu sein, führen realistische Attributionen eher zu funktionalem und unrealistische Attributionen eher zu dysfunktionalem leistungsbezogenem Verhalten (Försterling, 2000; Försterling \& Morgenstern, 2002). Während Personen mit realistischen Attributionen Zeit in Gebiete investieren, in denen sie Stärken aufweisen, vergeuden Personen mit unrealistischen Attributionen Zeit mit Aufgaben, die sie aufgrund mangelnder Fähigkeiten nicht oder kaum schaffen können.

\section{Sportwiss $2011 \cdot 41: 190-201 \quad$ DOI 10.1007/s12662-011-0195-7}

(c) Springer-Verlag 2011

\section{Mirko Schmidt · Achim Conzelmann \\ Selbstkonzeptförderung im Sportunterricht. Eine psychologische Betrachtung einer pädagogischen Zielperspektive}

\section{Zusammenfassung}

In schulsportbezogenen Interventionsstudien, welche eine Überprüfung der Sozialisationshypothese zum Ziel hatten, wurde bis anhin versucht, das Selbstkonzept der Schülerinnen und Schüler zu erhöhen, was mit Blick auf die Funktionalität fragwürdig erscheint. Denn sowohl aus pädagogischer als auch aus psychologischer Perspektive ist zu fragen, ob nicht eher eine möglichst realitätsangemessene Ausprägung des Selbstkonzepts anzustreben ist. So dürfte eine Unterschätzung leistungsbezogenes Verhalten hemmen und eine Überschätzung kann ins-

besondere in sozialen Interaktionen dysfunktional sein. Ausgehend von diesem Widerspruch wird im folgenden Beitrag die Frage behandelt, welche Selbstkonzeptausprägungen aus einer psychologischen Perspektive durch den Sportunterricht gefördert werden sollen. Dabei entsteht eine neue Zielperspektive für zukünftige selbstkonzeptfördernde Interventionen im Sportunterricht.

\section{Schlüsselwörter}

Funktionalität · Selbsteinschätzung ·

Selbstkonzept · Sportunterricht · Veridikalität

\section{Promoting the self-concept in physical education. A psychological look at an educational goal}

\section{Abstract}

Previous intervention studies in school sports designed to test the socialisation hypothesis have tried to raise students' self-concepts. However, this goal seems to be of questionable functionality: From both an educational and a psychological perspective, it would seem preferable to aim toward making selfconcepts as realistic as possible. Underestimating self-concepts might impede performance-related behaviour, whereas overestimating might be dysfunctional, particularly in social interactions. This dichotomy is used to examine which manifestations of the selfconcept physical education needs to promote from a psychological perspective. It creates a new perspective for future interventions designed to promote the self-concept in physical education.

\section{Keywords}

Functionality · Self-assessment .

Self-concept · Physical education · Veridicality 


\section{Selbstunterschätzung}

Motivationspsychologische Studien untersuchen neben der realistischen Einschätzung und der Überschätzung auch die Unterschätzung der eigenen Fähigkeiten und kommen zumindest in Bezug auf letztere zu einem einheitlichen Schluss (Weiss \& Ferrer-Caja, 2002, S. 112-114). So zeigt sich beispielsweise in einer Studie von Weiss und Horn (1990), dass Überschätzer, Realisten und Unterschätzer unterschiedliche leistungsbezogene Persönlichkeitsmerkmale aufweisen. Aufgrund von Erhebungen der selbsteingeschätzten sportlichen Fähigkeit, der erhobenen sportmotorischen Kompetenz und den daraus gebildeten Differenzwerten, teilten sie die Kinder in 3 Gruppen ein: solche, die ihre Fähigkeiten unterschätzten, solche, die sich realistisch einschätzten und solche, die sich überschätzten. Die Ergebnisse zeigten, dass Mädchen, die sich unterschätzten, weniger Leistungsmotivation, höhere Leistungsangst und eher externale Kontrollüberzeugungen aufwiesen als Mädchen, die sich realistisch einschätzten. Sich unterschätzende Jungen unterschieden sich nur in Bezug auf die Kontrollüberzeugungen von den Überschätzern und den Realisten. Phillips und Zimmerman (1990) haben (bzgl. akademischer Fähigkeiten) hochbegabte Schüler untersucht, welche ihre schulischen Fähigkeiten stark unterschätzen. Dabei haben sie herausgefunden, dass diejenigen, die diese ,illusion of incompetence" aufwiesen, gleichzeitig auch unrealistisch tiefe Erfolgserwartungen und hohe Leistungsangst zeigten sowie selten Aufgaben mit adäquatem Schwierigkeitsgrad wählten. Das Problem inadäquater Aufgabenwahl von Über- und Unterschätzern thematisiert auch Harter (1998, S. 590), die nach ähnlichen Befunden zum Schluss kommt, dass in einem motivationalen Kontext sowohl eine starke Über- als auch eine starke Unterschätzung als dysfunktional für den Erwerb neuer Fähigkeiten taxiert werden muss. Eine maßvolle Überschätzung erachtet sie für das Setting Schule als den funktionalsten Zustand, da dieser mit einer höheren Leistungsmotivation einhergehe als eine realistische Einschätzung, jedoch weniger negative Konsequenzen mit sich bringe als eine starke Über- oder Unterschätzung.

\section{Differenzierte Betrachtung des Problems}

Betrachtet man die Ergebnisse der berichteten Studien im Überblick, scheint die Befundlage weder eindeutig für eine Selbstüberschätzung noch für eine realistische Einschätzung der eigenen Fähigkeiten zu sprechen und bleibt somit weiterhin für empirische Untersuchungen offen. Einheitliche Befunde finden sich einzig in Bezug auf die Unterschätzung der eigenen Fähigkeiten, welche durchgehend als dysfunktionaler Zustand ermittelt wird. Dabei stellt sich die Frage, ob überhaupt von der Funktionalität gesprochen werden darf oder nicht eher von differenziellen und komplexen Effekten ausgegangen werden muss. Wie den referierten Befunden zu entnehmen ist, kann der funktionale Wert einer Über- oder Unterschätzung der eigenen Fähigkeiten in unterschiedlichen Situationen und unter verschiedenen Bedingungen erheblich variieren. Wenn Harter (1998, S. 590) eine leichte Überschätzung als optimalen Zustand im schulischen Setting und zum Erwerb neuer Fähigkeiten betont, spricht sie damit gleich zwei Aspekte an: Es gilt sowohl das Ausmaß der Verzerrung als auch den Kontext, in dem sich ein Individuum befindet (Situationsmerkmale), zu beachten. Nicht in jeder Situation wird eine realistische Selbsteinschätzung den gleichen Erfolg oder Misserfolg mit sich bringen. Während Taylor und Brown (1988) einer Überschätzung kurzfristig funktionalen Wert für das psychische Wohlbefinden (internales Kriterium) zuschreiben, sehen Anderson et al. (2006) langfristig eher negative Konsequenzen für das sich überschätzende Individuum im sozialen Gefüge (externales Kriterium). Welches Kriterium zur Beantwortung der Funktionalitätsfrage herangezogen wird, ist also von entscheidender Bedeutung (Kriteriumsfrage). Und nicht zuletzt ist zu fragen, ob beispielsweise eine Überschätzung für jede Person den gleichen funktionalen Wert besitzt. So ist durchaus vorstellbar, dass weitere Persönlichkeits- merkmale wie Extraversion oder Offenheit für Erfahrungen die Funktionalität der Überschätzung moderieren könnten, was eine differenzielle Perspektive erforderlich machen würde. Vor diesem Hintergrund scheint eine differenziertere Betrachtung dieser vier genannten Faktoren von Nöten zu sein, um aus ihnen eine psychologisch orientierte Zielperspektive für eine selbstkonzeptfördernde Schulsportintervention ableiten zu können.

\section{Ausmaß der Verzerrung}

Die Frage nach der Bedeutung von Selbstüberschätzungstendenzen oder illusionär positiven Denkens für die Gesundheit und eine erfolgreiche Entwicklung wird ausführlich von Baumeister (1989) diskutiert. Seiner Meinung nach kann eine allzu optimistische Selbstsicht besonders negative Folgen haben und sollte deshalb vermieden werden. Eigene Fähigkeiten werden über-, Risiken unterschätzt. Er warnt aber nicht nur vor den negativen Konsequenzen, sondern schlägt in gewissem Maße eine konstruktive Lösung der etwas dichotom geführten Optimismus-Realismus-Debatte vor, indem er einen optimalen Bereich des Ausmaßes positiver Illusionen („optimal margin of illusion") postuliert, der mit einem optimalen psychologischen Funktionieren einhergehen soll. Dieser Bereich, der in einer leichten Überschätzung der eigenen Fähigkeiten liegt, ermöglicht es dem Individuum, die Vorzüge des illusionär optimistischen und des realistischen Denkens zu verbinden. Abweichungen von diesem angeblich optimalen Bereich in Richtung einer realitätsfernen Selbstüberschätzung sollten ihm zu Folge zu unproduktiver Persistenz oder $\mathrm{zu}$ anderen selbstbehindernden Strategien (z. B. Self-Handicapping, Tice, 1991) führen.

Die unproduktive Persistenz wurde von McFarlin, Baumeister und Blascovich (1984) erstmals untersucht und in weiteren Studien erfolgreich repliziert. Bedingt durch die Überschätzung der eigenen Fähigkeiten unerreichbare Ziele über eine längere Zeit verfolgen oder unlösbare Aufgaben nicht bei Seite legen zu wollen, führt nicht nur zu schlechteren Gesamt- 
leistungen (Vancouver, Thompson, Tischner \& Putka, 2002), sondern auch zu negativen Konsequenzen für das psychische Wohlbefinden (Carver \& Scheier, 1998, S. 189) oder für die Gesundheit (Solberg Nes, Segerstrom \& Sephton, 2005). Dahingegen kann eine realistische Einschätzung der eigenen Fähigkeiten zu einer früheren Zielablösung führen und somit personelle Ressourcen - z. B. Zeit und Energie - frei machen, um erreichbare Ziele zu verfolgen (Wrosch, Scheier, Miller, Schulz \& Carver, 2003).

Die Strategie des Self-Handicappings kann besonders gut vor Leistungssituationen beobachtet werden. So berichten beispielsweise im Bereich des Leistungssports Sportler vor wichtigen Wettkämpfen sehr offenkundig über Beschwerden, leichte Verletzungen oder unzureichendes Training, um sich somit gegen spätere Kritik an ihren Leistungen zu immunisieren (Mummendey, 2006, S. 82-84). Solche selbst auferlegten Handicaps ermöglichen dem Individuum attributionale Vorteile und können eine selbstwertschützende Wirkung haben: Bei Misserfolg kann das Versagen auf das Handicap attribuiert werden, bei Erfolg scheint die eigene, unter erschwerten Bedingungen erbrachte Leistung besonders bemerkenswert. Diese Attributionsstrategien steigern zwar kurzfristig den Selbstwert, haben langfristig aber sowohl auf letzteren als auch auf die Lern- und Leistungsmotivation sowie auf die zukünftige Leistung einen negativen Effekt (McCrea, 2008; McCrea \& Hirt, 2001; Urdan, 2004; Zuckerman, Kieffer \& Knee, 1998).

Auch wenn Baumeister (1989, S. 184185) zusätzlich noch den Fall der Unterschreitung des optimalen Bereichs positiver Illusionen diskutiert und dabei auf die demotivierenden und leistungshemmenden Auswirkungen der Unterschätzung eigener Fähigkeiten verweist (Harter, 1998, S. 590; Weiss \& Ferrer-Caja, 2002, S. 112-114), scheint das Problem noch nicht hinreichend gelöst. Die Frage, um wie viel die Realität ins Positive verzerrt sein soll, damit man sich noch im idealen Bereich befindet, dürfte erstens schwierig zu beantworten sein und zweitens von den jeweiligen individuellen und situativen Voraussetzungen abhängen.

\section{Situationsmerkmale}

Mit der Bedeutung situativer Gegebenheiten zur Bestimmung funktionaler Selbsteinschätzungen beschäftigt sich auch Bandura (1994). Er benennt Situationen, in denen illusionäre Selbstüberschätzung äußerst gefährlich sein kann. Wenn sich beispielsweise jemand zum Ziel gesetzt hat, schwimmend einen See $\mathrm{zu}$ überqueren und dabei seine schwimmerischen Fähigkeiten überschätzt, kann dies ebenso verheerende Konsequenzen haben, wie wenn man seine radfahrerischen Fähigkeiten im Straßenverkehr überschätzt.

"However, the functional value of accurate self-appraisal depends on the nature of the activity. Activities in which mistakes can produce costly or injurious consequences call for accurate self-appraisal of capabilities. It is a different matter where difficult accomplishments can produce substantial personal and social benefits and the costs involve one's time, effort, and expendable resources." (Bandura, 1994, S. 76)

Ebenso gibt es aber auch Situationen, in denen eine Überschätzung der eigenen Fähigkeiten und die damit einhergehenden motivationalen Effekte durchaus funktionalen Wert besitzen:

„When people err in their self-appraisal they tend to overestimate their capabilities. This is a benefit rather than a cognitive failing to be eradicated. If efficacy beliefs always reflected only what people can do routinely they would rarely fail but they would not set aspirations beyond their immediate reach nor mount the extra effort needed to surpass their ordinary performances." (Bandura, 1994, S. 76)

Empirische Studien scheinen die angedeutete Situationsspezifik zu bestätigen und darauf hinzuweisen, dass die Überschätzung besonders der physischen Fähigkeiten schwerwiegende Konsequenzen nach sich ziehen kann. Radfahrunfälle gehören laut einer Untersuchung von Rivara (1985) zu den häufigsten Ursachen schwerwiegender Verletzungen im Kindesalter. Nicht selten ist daran eine Fehleinschätzung der eigenen Fähigkeiten mitbeteiligt. So konnte Plumert (1995) in einer Studie an 6- und 8-Jährigen zeigen, dass eine Überschätzung der eigenen physischen Fähigkeiten mit der Anzahl und dem Schweregrad von Alltagsverletzungen zusammenhängt. Je stärker sich Kinder überschätzen, desto größer ist die Gefahr, sich zu verletzen. Geschlechtsspezifische Auswertungen ergaben, dass dieser Effekt bei den Jungen stärker war als bei den Mädchen. Da Ergebnisse weiterer Studien dieser Arbeitsgruppe in die gleiche Richtung weisen (Plumert, Kearney \& Cremer, 2004; Plumert \& Schwebel, 1997; Schwebel \& Bounds, 2003), warnen die Autoren besonders bei physischen oder sportlichen Betätigungen vor einer allzu starken Überschätzung der eigenen Fähigkeiten. Allerdings betonen sie auch, dass diese Resultate keinesfalls dazu veranlassen sollen, eine Unterschätzung als funktionalen Zustand zu proklamieren. Auch wenn die Unterschätzung der eigenen physischen Fähigkeiten Kinder vor Unfällen schützen kann, antizipieren Schwebel und Plumert (1999) ähnlich wie Bandura (1994) negative Konsequenzen für die von ihnen gefundene Gruppe der Unterschätzer: Denn wer sich unterschätzt, verpasst Gelegenheiten, die eigenen Fähigkeiten unter Beweis zu stellen und Neues zu lernen. Eine angemessene physische als auch psychische Entwicklung beinhaltet aber besonders im Kindes- und Jugendalter, Neues auszuprobieren und daran zu wachsen (Bjorklund \& Green, 1992). Während eine Überschätzung in gefährlichen Situationen das Unfallrisiko erhöhen kann, wird deren Funktionalität als Motor der Entwicklung in ungefährlichen oder beaufsichtigten Situationen durchaus zugestanden. Ohne die Berücksichtigung der jeweiligen Situation scheint eine sinnvolle Funktionalitätsbestimmung also kaum möglich zu sein.

Taylor und Brown (1994) wollen nicht nur unterschiedliche Situationen, sondern auch unterschiedliche Handlungsphasen und deren Bewusstseinslagen beachtet wissen, um funktionale Selbsteinschätzungen ausfindig machen zu können. Sie gehen davon aus, dass Individuen in der Lage sind, ihre positiven Illusionen aufzugeben, wenn eine bestimmte Situation eine realitätsangemessene Einschätzung erfordert. Durch dieses ,window of realism“ (S. 26) könne der Mensch trotz seiner tendenziell positiv illusionären Erwar- 
tungen lauernde Gefahren erkennen und entsprechend richtig handeln. Das einer Zielsetzung vorauslaufende Abwägen alternativer Zielsetzungen wäre nach ihnen eine Situation, in der sich eine realistische Selbsteinschätzung lohnt, um die eigenen Ressourcen gewinnbringend einzusetzen. Zur Überprüfung dieser Hypothese nutzten Taylor und Gollwitzer (1995) die Unterscheidung zwischen abwägender (prädezisionaler) und planender (postdezisionaler) Bewusstseinslage und versetzten ihre Probanden (alles Studierende) in die beiden unterschiedlichen Bewusstseinslagen. Danach wurden sie zur aktuellen Stimmungslage, zum Selbstwert und zu allgemeinen Risikoeinschätzungen befragt. Wie erwartet wiesen die Probanden in der abwägenden Bewusstseinslage niedrigere Werte in Bezug auf die Stimmung und den Selbstwert, aber höhere Werte bezüglich ihrer Risikoeinschätzungen auf. Bei den Probanden in der planenden Bewusstseinslage zeigte sich das entgegengesetzte Muster. In einer prädezisionalen Phase sollte folglich eine möglichst realistische Sicht der eigenen Kompetenzen und Risiken vorherrschen, um eine optimale Wahl des anzustrebenden Ziels zu ermöglichen. Ist das Ziel einmal festgelegt und die Entscheidung getroffen, scheinen die zuvor noch als hinderlich bestimmten positiven Verzerrungen motivierenden Charakter anzunehmen, was zu einer Erhöhung der Persistenz führen kann, die zur Zielerreichung notwendig ist. Erfolgreiche Personen sind dabei imstande, sich den situativen Anforderungen flexibel anzupassen. Erfordert die Situation eine realistische Einschätzung der eigenen Fähigkeiten (Abwägen), so wird diese erbracht. Geht es um die Umsetzung des Plans, geben sich diese Personen wieder den für die Bewältigung dieser Handlungsphase funktionalen positiven Illusionen hin. Je nach Handlungsphase, in der man sich zu einer gewissen Zeit befindet, ist die Funktionalität realistischer Selbsteinschätzung also anders zu bewerten.

\section{Kriteriumsfrage}

Auch wenn aus der weiter oben skizzierten Optimismus-Realismus-Debatte durch ihre starke Fokussierung auf Methodenfragen und die gegenseitige Ableh- nung des zugrunde liegenden Menschenbildes kaum eine eindeutige Lösung für das Problem funktionaler Selbsteinschätzungen zu erwarten ist, lässt sich aus ihr trotzdem eine entscheidende Erkenntnis ableiten: Die Operationalisierung sowohl der unabhängigen (UV) als auch der abhängigen Variable (AV) scheint die Beantwortung der Funktionalitätsfrage stark zu beeinflussen.

So kann die Veridikalitätsvariable (UV) einerseits dadurch gebildet werden, dass Selbsteinschätzungen mit Fremdeinschätzungen verglichen werden (siehe z. B. Kwan, John, Kenny, Bond \& Robins, 2004). Hierbei ist allerdings fraglich, ob Fremdeinschätzungen prinzipiell präziser sein können als Selbsteinschätzungen, da ein Beobachter nie mehr Erfahrungen oder Beobachtungsgrundlagen haben kann als die befragte Person selbst (Funder, 1995, S. 657). Andererseits können Selbsteinschätzungen auch objektiven Standards oder Tests gegenübergestellt werden (Dunning, Heath \& Suls, 2004, S. 71). Studien haben beispielsweise Selbsteinschätzungen akademischer Fähigkeiten von Studierenden mit ihren Testleistungen oder Noten am Ende des Semesters (Kurman \& Eshel, 1998; Robins \& Beer, 2001) oder sportliche Fähigkeitseinschätzungen mit sportmotorischen Testleistungen verglichen (Germain \& Hausenblas, 2006). Diese zweite Variante hat gegenüber der ersten den Vorteil, dass Verzerrungstendenzen auf Seite der Fremdeinschätzung weitgehend ausgeschlossen werden können. Somit ist die zweite Variante prinzipiell besser geeignet, Über- oder Unterschätzung bei Probanden ausfindig zu machen.

Bei einer differenzierteren Betrachtung der Operationalisierung der Funktionalität (AV) zeigt sich ein ähnliches Bild. So bestimmt nicht nur die Lokation des gewählten Zielkriteriums (internal vs. external) die Ergebnisse entsprechender Studien, sondern auch die zeitliche Perspektive, die zur Funktionalitätsbestimmung eingenommen wird. Studien, die internale Kriterien wie Wohlbefinden, Selbstwert oder Selbstwirksamkeitserwartungen verwenden, kommen vorwiegend zum Schluss, dass eine Überschätzung der eigenen Fähigkeiten funktional sei (Bonanno et al., 2002; Tay- lor \& Brown, 1988; Taylor et al., 2000). Studien, die hingegen Fremdratings wie soziale Akzeptanz oder Indikatoren der Leistungsentwicklung als externale Kriterien heranziehen, ermitteln eher eine realistische Selbsteinschätzung als funktionalen Zustand (Anderson et al., 2006; Colvin et al., 1995; Paulhus, 1998). Durch diese in der Optimismus-Realismus-Debatte unbeachtete Unterscheidung in der Lokation des Zielkriteriums lösen sich die vermeintlichen Divergenzen der zitierten Forschungsergebnisse weitestgehend auf. Positive Sichtweisen der eigenen Person können für das Individuum günstig sein, während sich dieselbe Sichtweise durch entsprechende Handlungen in einem interpersonalen Bereich negativ auswirken kann. Das aus der Sozialpsychologie bekannte Phänomen der Illusion der Gruppenproduktivität (Stroebe, Diehl \& Abakoumkin, 1992) zeigt beispielhaft mögliche problematische Situationen auf, die im Anschluss an Gruppenarbeiten entstehen können. Der ungerechtfertigte Glaube, einen größeren Beitrag zu einer gelungenen Gruppenarbeit geleistet zu haben als alle anderen Beteiligten, wirkt sich kurzfristig positiv auf das Wohlbefinden des Individuums aus - und solange dieser Glaube unausgesprochen bleibt, scheint nichts dagegen zu sprechen, sich dieser Illusion hinzugeben. Sobald dieser Gedanke aber geäußert wird, kann dadurch ein Konflikt entstehen, der negative soziale Konsequenzen nach sich ziehen kann. Ebenso bedeutsam scheint die zeitliche Perspektive zu sein. Während Studien, die kurzfristige Konsequenzen betrachten, vorwiegend für eine Überschätzung der eigenen Fähigkeiten plädieren (z. B. Armor \& Taylor, 1998; Taylor \& Brown, 1994; Taylor \& Gollwitzer, 1995), bewerten Studien, die langfristige Effekte untersuchen, eher realistische Selbsteinschätzungen als funktional (Colvin et al., 1995; Paulhus, 1998; Robins \& Beer, 2001). Da sowohl internale und externale Kriterien als auch kurz- und langfristige Konsequenzen zur Operationalisierung der Funktionalität herangezogen werden und ihre Berechtigung haben können, ist für jede Studie a priori festzulegen, weshalb sich welche Operationalisierung als geeignet erweisen soll. 


\section{Differenzielle Perspektive}

Bei der Diskussion um die Funktionalität von realitätsnahen bzw. -fernen Selbsteinschätzungen blieb ein wesentlicher Aspekt bisher weitgehend unberücksichtigt: dass bestimmte Handlungsempfehlungen nicht für jedermann in gleichem Maße gelten können und sollen. Unterschiedliche Fähigkeiten und Persönlichkeitsmerkmale müssten prinzipiell ebenso in die Funktionalitätsbestimmung einfließen, wie die Situation oder die soziale Umwelt, in der sich jemand bewegt.

Ein differenzieller Zugang stellt beispielsweise die Unterscheidung zwischen strategischen Optimisten - welche die eigenen Fähigkeiten überschätzen - und defensiven Pessimisten - welche mögliche Gefahren überschätzen dar (Norem \& Illingworth, 2004). Hierbei handelt es sich um unterschiedliche Strategien, wie Individuen mit anstehenden Herausforderungen umgehen. Verschiedene Untersuchungen zeigen, dass eine Gegenüberstellung dieser beiden Coping-Strategien ohne Berücksichti- gung interindividueller Differenzen unsinnig ist und sich keine allgemeingültigen Handlungsempfehlungen ableiten lassen. So schneiden beide Gruppen in standardisierten Testsituationen etwa gleich gut ab, wenn sie sich entsprechend ihrer Coping-Strategie darauf vorbereiten können (Norem \& Illingworth, 1993; Tomaya, 2005). Während sich die strategischen Optimisten während der Vorbereitungszeit bewusst ablenken und sich auf vergangene ähnliche Situationen besinnen, in denen sie die Aufgabe erfolgreich bewältigt haben, malen sich defensive Pessimisten mögliche Szenarien aus, die sich aus einer Situation ergeben können, damit sie gedanklich auf alle Eventualitäten vorbereitet sind. Haben letztere nicht genügend Zeit zur Vorbereitung, werden sie nervös und erbringen verhältnismäßig schlechtere Leistungen. Dahingegen lassen sich strategische Optimisten verunsichern, wenn sie dazu angehalten werden, intensiv über eine zukünftige Leistungssituation nachzudenken. Hier muss sich eine Funktionalitätsbestimmung an den jeweiligen Fähigkei- ten und Dispositionen orientieren, also differenziell ausgerichtet sein.

Augenscheinlich wird die Notwendigkeit einer differenziellen Perspektive bei einer näheren Betrachtung der Studien zum Unfallverhalten von Kindern. Seit Längerem ist bekannt, dass Verhaltensstörungen (z. B. Aufmerksamkeitsdefizit-Hyperaktivitäts-Störung, ADHS; Störung mit oppositionellem Trotzverhalten, SOT) oder Persönlichkeits- und Temperamentseigenschaften (Extraversion; Sensation-Seeking) mit Unfällen von Kindern in Zusammenhang stehen (Manheimer \& Mellinger, 1967). Während extravertierte, sich überschätzende oder Sensation suchende Kinder im Verlauf ihrer Kindheit eher Verletzungen erleiden und in Verkehrsunfälle verwickelt sind, bleiben introvertierte, sich unterschätzende, ängstliche und folgsame Kinder eher unversehrt (Dal Santo, Goodman, Glik \& Jackson, 2004; Halberschmidt, 2008, S. 62-68; Morrongiello \& Sedore, 2005; Plumert, 1995; Schwebel \& Plumert, 1999; Schwebel, 2004). Ist man nun für die Unversehrtheit von Kindern verantwortlich, die

\section{Hier steht eine Anzeige.}

\section{Springer}


sich durch hohe Sensation-Seeking-Werte und eine Überschätzung der eigenen Fähigkeiten auszeichnen, wird man voraussichtlich anders Handeln als wenn man es mit sich unterschätzenden, eher ängstlichen Kindern zu tun hat.

\section{Konsequenzen für selbstkon- zeptfördernden Sportunterricht}

Welche Selbstkonzeptausprägungen sollen nun aus einer psychologischen Perspektive durch den Sportunterricht gefördert werden? Die theoretischen Überlegungen und die Analyse empirischer Studien zum Thema funktionaler Selbsteinschätzungen erbrachten die Erkenntnis, dass die Suche nach einer eindeutigen und für alle Situationen und Personen gültige Funktionalitätsbestimmung notwendigerweise ins Leere gehen muss. Ohne die Berücksichtigung personeller und situativer Begebenheiten kann weder ein optimales Ausmaß an Verzerrungstendenzen bestimmt noch eine geeignete Operationalisierung empfohlen werden. Wenn es im Folgenden also darum geht, Konsequenzen für eine selbstkonzeptfördernde Intervention abzuleiten, müssen diese vier ausgeführten Faktoren Berücksichtigung finden.

Beginnend mit der Frage nach dem optimalen Ausmaß positiver Verzerrungen lässt sich aus den bisherigen Ausführungen eine Empfehlung höchstens dahingehend formulieren, dass für den Sportunterricht eine realistische Selbsteinschätzung bis maßvolle Überschätzung der eigenen Fähigkeiten - im Sinne eines veridikalen bis leicht „positiv gefärbten Selbstkonzepts“ (Sygusch, 2008, S. 143) - die ideale Zielperspektive darstellt. Denn eine Unterschätzung dürfte sich sowohl kurz- als auch langfristig negativ auf die motivationale und affektive Entwicklung auswirken (Harter, 1998, S. 590; Weiss \& Ferrer-Caja, 2002, S. 112114) und eine starke Überschätzung der eigenen Fähigkeiten kann gerade bei risikoreichen sportlichen Aktivitäten zu schwerwiegenden Unfällen oder Verletzungen führen. Durch den eruierten $\mathrm{Zu}$ sammenhang zwischen Unfallgefahr und bestimmten Temperaments- und Persönlichkeitseigenschaften wird die Bedeutung einer differenziellen Perspektive an dieser
Stelle besonders deutlich. So scheinen diejenigen Kinder, die sich sowohl überschätzen als auch hohe Extraversionswerte aufweisen, besonders oft in Verkehrsunfälle verwickelt zu sein und sich mehr Verletzungen zuzuziehen als andere (Plumert, 1995; Plumert \& Schwebel, 1997). Während extravertierte Überschätzer also auf ein realistisches Niveau gedrosselt werden sollten, dürften introvertierte Unterschätzer eher von Unterrichtsinszenierungen profitieren, die ihr Selbstkonzept zu erhöhen versuchen. Der Sportunterricht sollte folglich derart gestaltet sein, dass sowohl eigene Stärken als auch eigene Schwächen bewusst gemacht werden können, wobei Überschätzer eher auf ihre Schwächen und Unterschätzer eher auf ihre Stärken fokussieren sollten. Die Lehrperson steht dabei vor der anspruchsvollen Aufgabe, einzelne Schülerinnen und Schüler in ihrem Selbstkonzept zu fördern, während sie andere, die sich um eine weitere Erhöhung des Selbstkonzepts bemühen, eher zu einer realistischen Selbsteinschätzung verhelfen sollte. Nur ein individualisierter Unterricht oder eine differenziell angelegte Intervention kann diesem Desiderat gerecht werden.

Die Situationsmerkmale müssen in mehrerlei Hinsicht Beachtung finden. So ist es nicht unerheblich, ob im Sportunterricht die Steigerung der Ausdauerleistungsfähigkeit auf der 40o-m-Rundbahn oder das Schwimmen in offenem Gewässer auf dem Programm steht. Je nach Gefahr, die einer bestimmten sportlichen Aktivität innewohnt, muss einer realistischen Einschätzung der eigenen Fähigkeiten mehr oder weniger Wert beigemessen werden. Eine weitere, nicht zu vernachlässigende situationale Bedingung ist die im Laufe der Entwicklung zunehmende Selbstbestimmung beim Lernen (Flammer, 1983, S. 221). Nicht nur bedingt durch die Pisa-Studie, sondern auch durch die stetig zunehmende Bedeutung des lebenslangen Lernens, ist der Ruf nach selbstgesteuerten Lernformen und Unterrichtskonzepten lauter geworden (Konrad $\&$ Traub, 2009). Die Bedeutung, die eine realistische Selbsteinschätzung für eine erfolgreiche Realisierung selbstgesteuerter oder selbstregulierter Lernprozesse einnimmt, ist offensichtlich. Im von Carver und Scheier (1981, S. 203-222) vorgelegten
Modell der Selbstregulation stellt die Einschätzung der eigenen Fähigkeiten den ersten Schritt des in Feedback-Schleifen verlaufenden Regelkreises dar. Nur eine präzise Kenntnis der eigenen Fähigkeiten führt zur Wahl eines erreichbaren Ziels, das mit Nachdruck und entsprechender Selbstmotivierung erreicht werden kann. Bringen wir diese Erkenntnis mit denjenigen der Handlungsphasen zusammen, zeichnet sich ein relativ deutliches Bild: Bevor eine Handlung initiiert wird, also in der prädezisionalen Phase, scheint eine realistische Selbsteinschätzung besonders funktional zu sein, während in der postdezisionalen Phase eine Überschätzung der eigenen Fähigkeiten hilft, die angefangene Aufgabe zu Ende zu führen (Taylor \& Gollwitzer, 1995, S. 224). Geht man nun davon aus, dass, um auf die immer rascher von statten gehenden Veränderungen von Qualifikations- und Arbeitsprozessen zu reagieren, in Zukunft selbstgesteuerte Lernformen weiter zunehmen werden, scheint die Empfehlung, auch im Sportunterricht auf eine realistische Einschätzung bis maßvolle Überschätzung der eigenen Fähigkeiten $\mathrm{zu}$ fokussieren und sich in dieser zu üben, durchaus angebracht.

Die genauere Analyse der Kriteriumsfrage hat zu Tage gebracht, dass die Wahl des Kriteriums die Funktionalitätsbestimmung in hohem Maße beeinflusst. Im Hinblick auf mögliche Auswirkungen auf den interpersonalen Bereich (externales Kriterium) kann die Empfehlung nur in Richtung einer veridikalen Selbsteinschätzung gehen. Da im Sportunterricht Gruppenaktivitäten schon aus Sportart konstituierenden Gesichtspunkten besonderen Stellenwert besitzen, muss dem zwischenmenschlichen Aspekt besondere Beachtung geschenkt werden. So werden in kaum einem anderen Schulfach der eigene Beitrag zum Gelingen einer Gruppenarbeit sowie die eigene Fähigkeit für alle Beteiligten so offenkundig und unmittelbar dargestellt wie im Sportunterricht. Überschätzer dürften sich also in diesem Setting kurzfristig Vorteile verschaffen und höhere soziale Akzep$\tan z$ genießen (Paulhus, 1998), langfristig aber mehr negative als positive Konsequenzen erfahren. Dass der eigene Beitrag zum gelungenen Sieg nicht dem einzelnen Überschätzer, sondern der gan- 
zen Mannschaft zu verdanken ist, werden früher oder später die meisten Mitschüler bemerken. Da bei Kindern und Jugendlichen die soziale Akzeptanz stark mit den physischen Fähigkeiten korreliert (Weiss \& Amorose, 2008, S. 126), ergibt sich auch für Unterschätzer ein Problem: Indem sie sich oft mit zu schwierigen oder zu leichten Aufgaben beschäftigen, bietet sich ihnen kaum die Gelegenheit, sich als kompetente Schüler hervorzutun, was sich wiederum in einer geringeren Akzeptanz durch die Peers widerspiegeln kann. Um also die soziale Akzeptanz für alle Schülerinnen und Schüler zu gewährleisten, sollte auch hier eine realistische Einschätzung der eigenen Fähigkeiten favorisiert werden. Die Wahrung sozialer Akzeptanz einer Person in der Gruppe ist schon deshalb von zentraler Bedeutung, weil sie eine wichtige Quelle des Selbstwerts (als internales Kriterium) darstellt (Schütz, 2003, S. 57-70) und sich somit sowohl aus einer kurz- als auch aus einer langfristigen Perspektive als ein erstrebenswertes Ziel erweist.

\section{Fazit}

In diesem Beitrag wurde versucht, eine neue Zielperspektive für selbstkonzeptfördernde Schulsportinterventionen $\mathrm{zu}$ etablieren. Es konnte gezeigt werden, dass in einem sportpädagogischen Kontext die alleinige Fokussierung auf die Höhe des Selbstkonzepts ohne die Berücksichtigung der Veridikalität aus psychologischer Sicht wenig Sinn macht und dass die Beantwortung der Funktionalitätsfrage einer differenzierten Betrachtung bedarf. Nach der Analyse des schulsportlichen Settings anhand der vier elaborierten Kriterien hat sich eine realistische Einschätzung bis maßvolle Überschätzung der eigenen Fähigkeiten in vielerlei Hinsicht als die funktionalste Selbstkonzeptausprägung herausgestellt.

Für zukünftige Forschung stellt sich die Aufgabe, empirisch zu prüfen, ob ein veridikales bis leicht erhöhtes Selbstkonzept im Sportunterricht tatsächlich den dargestellten funktionalen Wert besitzt: Sind Schülerinnen und Schüler mit einer realistischen Selbsteinschätzung motivierter und erzielen sie in der gleichen Zeit mehr Leistungsfortschritte als Über- und Un- terschätzer? Sind Realisten generell zufriedener mit sich und erfahren größere soziale Akzeptanz bei den Peers? Verletzen sie sich weniger?

Aus sportpädagogischer Sicht ist zudem zu fragen, wie dieses definierte Ziel erreicht werden kann. Die Befunde von Conzelmann, Schmidt und Valkanover (in Druck) zeigen, dass sich die intendierte Wirkung auf das Selbstkonzept von Schülerinnen und Schülern nicht durch den Sportunterricht per se einstellt. Vielmehr verlangt die Förderung funktionaler Selbstkonzepte eine spezifische Form der Inszenierung des Sportunterrichts, bei der insbesondere die psychologischen Wirkmechanismen der Selbstkonzeptgenese (z. B. Filipp \& Mayer, 2005) zu beachten sind.

\section{Korrespondenzadresse Mirko Schmidt}

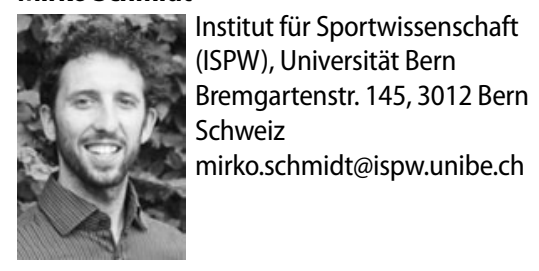

Interessenkonflikt. Der korrespondierende Autor gibt an, dass kein Interessenkonflikt besteht.

\section{Literatur}

1. Abramson, L.Y. \& Alloy, L.B. (1981). Depression, non-depression, and cognitive illusions: A reply to Schwartz. Journal of Experimental Psychology, 110, 436-447.

2. Allan, L.G., Siegel, S. \& Hannah, S. (2007). The sad truth about depressive realism. The Quarterly Journal of Experimental Psychology 60, 482495.

3. Alloy, L.B. \& Abramson, L.Y. (1979). Judgment of contingency in depressed and nondepressed students: Sadder but wiser? Journal of Experimental Psychology: General, 108, 441-485.

4. Anderson, C., Srivastava, S., Beer, J.S., Spataro, S. E. \& Chatman, J.A. (2006). Knowing your place: Self-perceptions of status in face-to-face groups. Journal of Personality and Social Psychology, 91, 1094-1110.

5. Annesi, J.J. (2007). Relations of age with changes in self-efficacy and physical self-concept in preadolescents participating in a physical activity intervention during afterschool care. Perceptual and Motor Skills, 105, 221-226.

6. Armor, D.A. \& Taylor, S.E. (1998). Situated optimism: Specific outcome expectancies and selfregulation. Advances in Experimental Social Psychology, 30, 309-379.

7. Bandura, A. (1994). Self-efficacy. In V. S. Ramachaudran (Ed.), Encyclopedia of human behavior (Vol. 4, pp. 71-81). New York: Academic Press.
8. Baumeister, R.F. (1989). The optimal margin of illusion. Journal of Social and Clinical Psychology, 8, 176-189.

9. Baumeister, R.F., Bushman, B.J. \& Campbell, W.K. (2000). Self-esteem, narcissism, and aggression: Does violence result from low self-esteem or from threatened egoism? Current Directions in Psychological Science, 9, 26-29.

10. Baumeister, R.F., Campbell, J.D., Krueger, J.I. \& Vohs, K.D. (2003). Does high self-esteem cause better performance, interpersonal success, happiness, or healthier lifestyles? Psychological Science in the Public Interest, 4, 1-44.

11. Bjorklund, D.F. \& Green, B.L. (1992). The adaptive nature of cognitive immaturity. American Psychologist, 47, 46-54.

12. Bonanno, G.A., Field, N.P., Kovacevic, A. \& Kaltman, S. (2002). Self-enhancement as a buffer against extreme adversity: Civil war in Bosnia and traumatic loss in the United States. Personality and Social Psychology Bulletin, 28, 184196.

13. Bonanno, G.A., Rennicke, C. \& Dekel, S. (2005). Self-enhancement among high-exposure survivors of the September 11 th terrorist attack: Resilience or social maladjustment? Journal of Personality and Social Psychology, 88, 984-998.

14. Bong, M. \& Skaalvik, E.M. (2003). Academic selfconcept and self-efficacy: How different are they really? Educational Psychology Review, 15, $1-40$.

15. Brettschneider, W.-D. \& Kleine, T. (2002). Jugendarbeit in Sportvereinen. Anspruch und Wirklichkeit. Schorndorf: Hofmann.

16. Brissette, I., Scheier, M.F. \& Carver, C. (2002). The role of optimism in social network development, coping, and psychological adjustment during a life transition. Journal of Personality and Social Psychology, 82, 102-111.

17. Burrmann, U. (2004). Effekte des Sporttreibens auf die Entwicklung des Selbstkonzepts Jugendlicher. Zeitschrift für Sportpsychologie, 11, 7182.

18. Bushman, B. \& Baumeister, R.F. (1998). Threatened egotism, narcissism, self-esteem, and direct and displaced aggression: Does self-love or selfhate lead to violence? Journal of Personality and Social Psychology, 75, 219-229.

19. Calfas, K. \& Cooper, D. (1996). Effect of a 5 week exercise training program on self-worth among adolescent girls: A randomised controlled study. Medicine and Science in Sports and Exercise, 28, 135.

20. Carson, R.C., Hollon, S.D. \& Shelton, R.C. (2010). Depressive realism and clinical depression. $B e-$ haviour Research and Therapy, 48, 257-265.

21. Carver, C.S. \& Scheier, M.F. (1981). Attention and self-regulation: A control-theory approach to human behavior. New York: Springer-Verlag.

22. Carver, C.S. \& Scheier, M.F. (1998). On the self-regulation of behavior. New York: Cambridge University Press.

23. Church, A.T., Katigbak, M.S., del Prado, A.M., Valdez-Medina, J.L., Miramontes, L.G. \& Ortiz, F.A. (2006). A cross-cultural study of trait self-enhancement, explanatory variables, and adjustment. Journal of Research in Personality, 40, 11691201.

24. Colvin, C.R. \& Block, J. (1994). Do positive illusions foster mental health? An examination of the Taylor and Brown formulation. Psychological Bulletin, 116, 3-20. 
25. Colvin, C.R., Block, J. \& Funder, D.C. (1995). Overly positive self-evaluations and personality: $\mathrm{Ne}$ gative implications for mental health. Journal of Personality and Social Psychology, 68, 11521162.

26. Conzelmann, A. (2001). Sport und Persönlichkeitsentwicklung: Möglichkeiten und Grenzen von Lebenslaufanalysen. Schorndorf: Hofmann.

27. Conzelmann, A. (2009). Differentielle Sportpsychologie - Sport und Persönlichkeit. In W. Schlicht \& B. Strauss (Hrsg.), Enzyklopädie der Psychologie: Serie V: Sportpsychologie, Band 1. Grundlagen der Sportpsychologie (S. 375-439). Göttingen: Hogrefe.

28. Conzelmann, A., Schmidt, M. \& Valkanover, S. (in Druck). Persönlichkeitsentwicklung durch Schulsport. Theorie, Empirie und Praxisbausteine der Berner Interventionsstudie Schulsport (BISS). Bern: Huber.

29. Costa, P.T. \& McCrae, R.R. (1992). Revised NEO Personality Inventory (NEO PI-R) and NEO Five Factor Inventory (NEO-FFI). Odessa: Psychological Assessment Resources.

30. Dal Santo, J.A., Goodman, R.M., Glik, D. \& Jackson, K. (2004). Childhood unintentional injuries: Factors predicting injury risk among preschoolers. Journal of Pediatric Psychology, 29, 273283.

31. Deutsche Vereinigung für Sportwissenschaft (dvs), Deutscher Sportlehrerverband (DSLV) \& Deutscher Olympischer Sportbund (DOSB) (2009). Memorandum zum Schulsport. Frankfurt am Main: Deutscher Olympischer Sportbund.

32. Dunning, D., Heath, C. \& Suls, J.M. (2004). Flawed self-assessment: Implications for health, education, and the workplace. Psychological Science in the Public Interest, 5, 69-106.

33. Filipp, S.-H. \& Mayer, A.-K. (2005). SelbstkonzeptEntwicklung. In J. B. Asendorpf (Hrsg.), Enzyklopädie der Psychologie. Entwicklungspsychologie, Band 3. Soziale, emotionale und Persönlichkeitsentwicklung (S. 259-334). Göttingen: Hogrefe.

34. Flammer, A. (1983). Die individuelle Steuerung kognitiver Aufbauprozesse. In L. Montada, K. Reusser \& G. Steiner (Hrsg.), Kognition und Handeln (S. 219-228). Stuttgart: Klett.

35. Försterling, F. (2000). Die Rolle vorauslaufender Bedingungen in attributionalen Verhaltensmodellen. In F. Försterling, J. Stiensmeier-Pelster \& L.-M. Silny (Hrsg.), Kognitive und emotionale Aspekte der Motivation (S. 29-52). Göttingen: Hogrefe.

36. Försterling, F. \& Morgenstern, M. (2002). Accuracy of self-assessment and task performance: Does it pay to know the truth? Journal of Educational Psychology, 94, 576-585.

37. Funder, D.C. (1995). On the accuracy of personality judgment: A realistic approach. Psychological Review, 102, 652-670.

38. Gerlach, E. (2008). Sportengagement und Persönlichkeitsentwicklung: eine längsschnittliche Analyse der Bedeutung sozialer Faktoren für das Selbstkonzept von Heranwachsenden. Aachen: Meyer \& Meyer.

39. Germain, J.L. \& Hausenblas, H.A. (2006). The relationship between perceived and actual physical fitness: A meta-analysis. Journal of Applied Sport Psychology, 18, 283-296.

40. Goni, A. \& Zulaika, L. (2000). Relationships between physical education classes and the enhancement of fifth grade pupils' self-concept. Perceptual and Motor Skills, 91, 246-250.
41. Gresham, F.M., Lane, K.L., MacMillan, D.L., Bocian, K.M. \& Ward, S.L. (2000). Effects of positive and negative illusory biases: Comparisons across social and academic self-concept domains. Journal of School Psychology, 38, 151-175.

42. Greve, W. (2000). Die Psychologie des Selbst Konturen eines Forschungsthemas. In W. Greve (Hrsg.), Psychologie des Selbst (S. 15-36). Weinheim: Beltz.

43. Halberschmidt, B. (2008). Psychologische Schulsport-Unfallforschung. Forum Sportwissenschaft, Band 16. Hamburg: Czwalina.

44. Harter, S. (1998). The development of self-representations. In W. Damon \& N. Eisenberg (Eds.), Handbook of child psychology: Vol 3. Social, emotional and personality development (5th ed., pp. 553-617). New York: John Wiley \& Sons.

45. Heim, R. (2002). Jugendliche Sozialisation und Selbstkonzeptentwicklung im Hochleistungssport. Eine empirische Studie aus pädagogischer Perspektive. Aachen: Meyer \& Meyer.

46. Helmke, A. (1992). Selbstvertrauen und schulische Leistungen. Göttingen: Hogrefe.

47. Hurrelmann, K. (2006). Einführung in die Sozialisationstheorie (9. Aufl.). Weinheim: Beltz.

48. Judge, T.A. \& Bono, J.E. (2001). Relationship of core self-evaluations traits - self-esteem, generalized self-efficacy, locus of control, and emotional stability - with job satisfaction and job performance: A meta-analysis. Journal of Applied Psychology, 86, 80-92.

49. Konrad, K. \& Traub, S. (2009). Selbstgesteuertes Lernen. Grundwissen und Tipps für die Praxis. Hohengehren: Schneider.

50. Krampen, G. (2002). Persönlichkeits- und Selbstkonzeptentwicklung. In R. Oerter \& L. Montada (Hrsg.), Entwicklungspsychologie (5. Aufl., S. 675-710). Weinheim: Beltz.

51. Kurman, J. \& Eshel, Y. (1998). Self-enhancement, generality level of self-evaluation, and emotional adjustment. Journal of Social Psychology, 138, 549-563.

52. Kurt, A. \& Paulhus, D.L. (2008). Moderators of the adaptiveness of self-enhancement: Operationalization, motivational domain, adjustment facet, and evaluator. Journal of Research in Personality, 42, 839-853.

53. Kwan, V.S., John, O.P., Kenny, D.A., Bond, M.H. \& Robins, R.W. (2004). Reconceptualizing individual differences in self-enhancement bias: An interpersonal approach. Psychological Review, 111, 94-110.

54. Kwan, V.S., John, O.P., Robins, R.W. \& Kuang, L.L. (2008). Conceptualizing and assessing self-enhancement bias: A componential approach. Journal of Personality and Social Psychology, 94, 1062-1077.

55. Lintunen, T. (1999). Development of self-perceptions during the school years. In Y. V. Auweele, F. Bakker, S. Biddle, M. Durand \& R. Seiler (Eds.), Psychology for physical educators (pp. 115134). Champaign, IL: Human Kinetics.

56. Lloyd, J. \& Fox, K.R. (1992). Achievement goals and motivation to exercise in adolescent girls: $A$ preliminary investigation study. British Journal of Physical Education Research Supplement, 11, 12-16.

57. Manheimer, D.I. \& Mellinger, G.D. (1967). Personality characteristics of the child accident repeater. Child Development, 38, 491-513.
58. Marsh, H.W., Byrne, B.M. \& Yeung, A.S. (1999). Causal ordering of academic self-concept and achievement: Reanalysis of a pioneering study and revised recommendations. Educational Psychologist, 34, 154-157.

59. Marsh, H.W. \& Hau, K.-T. (2003). Big-fish-littlepond-effect on academic self-concept: A crosscultural (26-country) test of the negative effects of academically selective schools. American Psychologist, 58, 364-376.

60. Marsh, H.W. \& Peart, N. (1988). Competitive and cooperative physical fitness training programs for girls: Effects on physical fitness and on multidimensional self-concepts. Journal of Sport and Exercise Psychology, 10, 390-407.

61. McCrea, S.M. (2008). Self-handicapping, excuse-making, and counterfactual thinking: Consequences for self-esteem and future motivation. Journal of Personality and Social Psychology, 95, 274-292.

62. McCrea, S.M. \& Hirt, E.R. (2001). The role of ability judgments in self-handicapping. Personality and Social Psychology Bulletin, 27, 1378-1389.

63. McFarlin, D.B., Baumeister, R.F. \& Blascovich, J. (1984). On knowing when to quit: Task failure, self-esteem, advice, and nonproductive persistence. Journal of Personality, 52, 138-155.

64. Montada, L. (2008). Fragen, Konzepte, Perspektiven. In R. Oerter \& L. Montada (Hrsg.), Entwicklungspsychologie (6., vollst. überarb. Aufl., S. 3-48). Weinheim: Beltz.

65. Morrongiello, B.A. \& Sedore, L. (2005). The influence of child attributes and social-situational context on school-age children's risk taking behaviors that can lead to injury. Journal of Applied Developmental Psychology, 26, 347-361.

66. Mummendey, H.D. (2006). Psychologie des "Selbst". Theorien, Methoden und Ergebnisse der Selbstkonzeptforschung. Göttingen: Hogrefe.

67. Neuber, N. (2007). Entwicklungsförderung im Jugendalter. Theoretische Grundlagen und empirische Befunde aus sportpädagogischer Perspektive. Schorndorf: Hofmann.

68. Norem, J.K. \& Illingworth, K.S. (1993). Strategydependent effects of reflecting on self and tasks: Some implications of optimism and defensive pessimism. Journal of Personality and Social Psychology, 65, 822-835.

69. Norem, J.K. \& Illingworth, K.S. (2004). Mood and performance among defensive pessimists and strategic optimists. Journal of Research in Personality, 38, 351-366.

70. Paulhus, D.L. (1998). Interpersonal and intrapsychic adaptiveness of trait self-enhancement: A mixed blessing? Journal of Personality and Social Psychology, 74, 1197-1208.

71. Percy, L.E., Dziuban, C.D. \& Martin, J.B. (1981). Analysis of effects of distance running on selfconcepts of elementary students. Perceptual and Motor Skills, 52, 42

72. Petrakis, E. \& Bahls, V. (1991). Relation of physical education to self-concept. Perceptual and Motor Skills, 73, 1027-1031.

73. Phillips, D.A. \& Zimmerman, M. (1990). The developmental course of perceived competence and incompetence among competent children. In R. J. Sternberg \& J. Kolligian (Eds.), Competence considered (pp. 41-66). New Haven, CT: Yale University Press.

74. Plumert, J.M. (1995). Relations between children's overestimation of their physical abilities and accident proneness. Developmental Psychology, 31, 866-876. 
75. Plumert, J.M., Kearney, J.K. \& Cremer, J.F. (2004). Children's perception of gap avoidances: Bicycling across traffic-filled intersections in an immersive virtual environment. Child Development, 75, 1243-1253.

76. Plumert, J.M. \& Schwebel, D.C. (1997). Social and temperamental influences on children's overestimation of their physical abilities: Links to accidental injuries. Journal of Experimental Child Psychology, 67, 317-337.

77. Prohl, R. (2006). Grundriss der Sportpädagogik (2. Aufl.). Wiebelsheim: Limpert.

78. Rivara, F.P. (1985). Traumatic deaths of children in the United States: Currently available prevention strategies. Pediatrics, 75, 456-462.

79. Robins, R.W. \& Beer, J.S. (2001). Positive illusion about the self: Short-term benefits and longterm costs. Journal of Personality and Social Psychology, 80, 340-352.

80. Robins, R.W. \& John, O.P. (1997). The quest for self-insight. Theory and research on accuracy and bias in self-perception. In R. Hogan, J. Johnson \& S. Briggs (Eds.), Handbook of Personality Psychology (pp. 649-679). New York: Academic Press.

81. Roebers, C.M. (2007). Entwicklung des Selbstkonzeptes. In M. Hasselhorn \& W. Schneider (Hrsg.), Handbuch der Entwicklungspsychologie (S. 381-391). Göttingen: Hogrefe.

82. Rogers, C.R. (1951). Client-centered therapy. Boston: Houghton Mifflin.

83. Schrader, F.-W. (2006). Diagnostische Kompetenz von Eltern und Lehrern. In D. H. Rost (Hrsg.), Handwörterbuch Pädagogische Psychologie (S. 95-100). Weinheim: Belz.

84. Schütz, A. (2003). Psychologie des Selbstwertgefühls. Von Selbstakzeptanz bis Arroganz (2. Aufl.). Stuttgart: Kohlhammer.

85. Schütz, A. \& Sellin, I. (2003). Selbst und Informationsverarbeitung. Zeitschrift für Differentielle und Diagnostische Psychologie, 24, 151-161.

86. Schwebel, D.C. (2004). Temperamental risk factors for children's unintentional injury: The role of impulsivity and inhibitory control. Personality and Individual Differences, 37, 567-578.

87. Schwebel, D.C. \& Bounds, M.L. (2003). The role of parents and temperament on children's estimation of physical ability: Links to unintentional injury prevention. Journal of Pediatric Psychology, 28, 505-516.

88. Schwebel, D.C. \& Plumert, J.M. (1999). Longitudinal and concurrent relations among temperament, ability estimation, and injury proneness. Child Development, 70, 700-712.

89. Sedikides, C., Rudich, E.A., Gregg, A.P., Kumashiro, M. \& Rusbult, C. (2004). Are normal narcissists psychologically healthy? Self-esteem matters. Journal of Personality and Social Psychology, $87,400-416$.

90. Shedler, J., Mayman, M. \& Manis, M. (1993). The illusion of mental health. American Psychologist, 48, 1117-1131.

91. Smith, T.L. (1982). Self-concepts and movement skills of third grade children after physical education programs. Perceptual and Motor Skills, $54,1145-1146$.

92. Solberg Nes, L., Segerstrom, S.C. \& Sephton, S.E. (2005). Engagement and arousal: Optimism's effects during a brief stressor. Personality and Social Psychology Bulletin, 31, 111-120.
93. Stiller, J. \& Alfermann, D. (2007). Promotion of a healthy self-concept. In J. Liukkonen, Y. V. Auweele, B. Vereijken, D. Alfermann \&Y. Theodorakis (Eds.), Psychology for physical educators. Student in Focus (Vol. 2, pp. 123-140). Champaign: Human Kinetics.

94. Stroebe, W., Diehl, M. \& Abakoumkin, G. (1992). The illusion of group effectivity. Personality and Social Psychology Bulletin, 18, 643-650.

95. Sygusch, R. (2007). Psychosoziale Ressourcen im Sport. Ein sportartenorientiertes Förderkonzept für Schule und Verein. Schorndorf: Hofmann.

96. Sygusch, R. (2008). Selbstkonzeptförderung im Jugendsport - Zufall oder zielgerichtet? In A. Conzelmann \& F. Hänsel (Hrsg.), Sport und Selbstkonzept. Struktur, Dynamik und Entwicklung (S. 140-156). Schorndorf: Hofmann.

97. Taylor, S.E. \& Brown, J.D. (1988). Illusion and wellbeing: A social psychological perspective on mental health. Psychological Bulletin, 103, 193210.

98. Taylor, S.E. \& Brown, J.D. (1994). Positive illusions and well-being revisited: Separating fact from fiction. Psychological Bulletin, 116, 21-27.

99. Taylor, S.E. \& Gollwitzer, P.M. (1995). Effects of mindset on positive illusions. Journal of Personality and Social Psychology, 69, 213-226.

100. Taylor, S.E., Kemeny, M.E., Reed, G.M., Bower, J.E. \& Gruenewald, T.L. (2000). Psychological resources, positive illusions, and health. American Psychologist, 55, 99-109.

101. Taylor, S.E., Lerner, J.S., Sherman, D.K., Sage, R.M. \& McDowell, N.K. (2003). Portrait of the self-enhancer: Well adjusted and well liked or maladjusted and friendless? Journal of Personality and Social Psychology, 84, 165-176.

102. Tice, D.M. (1991). Esteem protection or enhancement? Self-handicapping motives and attributions differ by trait self-esteem. Personality and Individual Differences, 60, 711-725.

103. Tietjens, M. (2009). Physisches Selbstkonzept im Sport. Hamburg: Czwalina.

104. Tomaya, M. (2005). Influence of cognitive strategies on test coping strategies and academic achievement: Defensive pessimism and strategic optimism. The Japanese Journal of Educational Psychology, 53, 220-229.

105. Urdan, T. (2004). Predictors of academic self-handicapping and achievement: Examining achievement goals, classroom goal structures, and culture. Journal of Educational Psychology, 96, 251-264.

106. Vancouver, J.B., Thompson, C.M., Tischner, E.C \& Putka, D.J. (2002). Two studies examining the negative effect of self-efficacy on performance. Journal of Applied Psychology, 87, 506-516.

107. Weiss, M.R. \& Amorose, A.J. (2008). Motivational orientations and sport behavior. In T. S. Horn (Ed.), Advances in Sport Psychology (3rd ed., pp. 115-155). Champaign, IL: Human Kinetics.

108. Weiss, M.R. \& Ferrer-Caja, E. (2002). Motivational orientations and sport behavior. In T. S. Horn (Ed.), Advances in sport psychology (pp. 101183). Champaign, IL: Human Kinetics.

109. Weiss, M.R. \& Horn, T.S. (1990). The relationship between children's accuracy estimates of their physical competence and achievement-related behaviors. Research Quarterly for Exercise and Sport, 61, 250-258.

110. Willard, G. \& Gramzow, R.H. (2009). Beyond oversights, lies, and pies in the sky: Exaggeration as goal projection. Personality and Social Psychology Bulletin, 35, 477-492.
111. Wilson, T.D. (2009). Know thyself. Perspectives on Psychological Science, 4, 384-389.

112. Wright, S.S. (2000). Looking at the self in a rosecolored mirror: Unrealistically positive self-views and academic performance. Journal of Social and Clinical Psychology, 19, 451-462.

113. Wrosch, C., Scheier, M.F., Miller, G.E., Schulz, R. \& Carver, C.S. (2003). Adaptive self-regulation of unattainable goals: Goal disengagement, goal reengagement, and subjective well-being. Personality and Social Psychology Bulletin, 29, 1494-1508.

114. Zuckerman, M., Kieffer, S.C. \& Knee, C.R. (1998). Consequences of self-handicapping: Effects on coping, academic performance, and adjustment. Journal of Personality and Social Psychology, $74,1619-1628$ 